\title{
Nuclear Sequestration of Virion Capsids in PML Cages is a Cellular Defense Against a Neurotropic Herpesvirus.
}

\author{
M. Reichelt,* M. Sommer,* L. Wang,* J. Perrino, ${ }^{* *}$ N. Sen, ${ }^{*}$ A. Nour,* L. Zerboni,* and A.M. \\ Arvin* \\ * Departments of Pediatrics and Microbiology \& Immunology, Stanford University School of \\ Medicine, Stanford CA 94305 \\ ** Cell Sciences Imaging Facility, Stanford University School of Medicine, Stanford CA 94305
}

Varicella-zoster virus (VZV) is a ubiquitous human alphaherpesvirus that causes varicella (chickenpox) during primary infection, can establish latency in sensory nerve ganglia and may reactivate causing herpes zoster (shingles). Although vaccines against chickenpox and shingles have been approved, VZV infection and reactivation still causes significant morbidity in immunodeficient people, cancer patients and the elderly [1]. New therapeutic strategies to reduce VZV pathogenesis could be developed by identification and modulation of host intrinsic immune factors that control virus reactivation, replication and spread.

VZV genome replication and packaging into virion nucleocapsids occurs in the cell nucleus. The viral ORF23 capsid protein is believed to decorate the outer surface of the nucleocapsid shell on hexons formed by the major capsid protein ORF40 [2]. Mature nucleocapsids need to traverse the nuclear membrane and undergo envelopment in the Golgi compartment before their release as infectious virus particles. Induction of nuclear sequestration or degradation of mature nucleocapsids could therefore limit their transfer to the cytoplasm and may be exploited to support antiviral therapy. Promyelocytic leukemia nuclear bodies (PML-NBs) are subnuclear compartments that promote the sequestration of neurotoxic proteins in some neurologic diseases like Spinocerebellar ataxia or Huntington disease and also contribute to host innate immunity against several DNA and RNA viruses [3, 4].

Here we investigated whether PML-NBs are capable of sequestering VZV nucleocapsids within the infected cell nucleus and contribute to the host antiviral defense against VZV. We identified by immuno-electron microscopy for the first time endogenous PML-NBs that sequester completely assembled VZV nucleocapsids in vitro and in neurons and satellite cells within human sensory ganglion (DRG) xenografts in vivo. Furthermore, we show for the first time that a specific PML isoform (PML-IV) uniquely promotes the sequestration of capsids within spherical PML-IV "cages". Quantitative immuno-EM revealed that nuclear PML-IV upregulation resulted in the entrapment of more than $95 \%$ of all nucleocapsids within PML-IV cages. Biochemical experiments revealed the molecular interaction of PML-IV with the outer VZV capsid protein ORF23, thus suggesting that this interactions contributes to the recognition and sequestration of capsids by PML-IV. Most importantly, viral titration experiments showed that wild-type PML-IV, but not a PML-IV deletion mutant deficient in capsid sequestration, inhibited the production of infectious VZV and resulted in a $50 \%$ reduction of viral titers. 
We conclude that VZV capsid sequestration in nuclear PML-IV cages correlates with PML-IV antiviral activity against VZV. Our results indicate that PML-mediated nuclear containment of neurotoxic proteins in some neurological diseases or of viral capsids upon VZV infection is a conserved cellular defense function that also restricts the neuropathogenic potential of neurotropic viruses like VZV. Furthermore, we identify PML-NBs as a therapeutic target for VZV-antiviral intervention and suggest that therapeutic stimulation of PML-mediated sequestration of VZV nucleocapsids may be used to support VZV antiviral therapy.

\section{References}

[1] J. I. Cohen et al, (2007). Varicella-Zoster Virus. In Fields Virology, P. M. H. David M. Knipe (Ed.), Philadelphia: Lippincott Williams and Wilkins, a Wolters Kluwer Business.

[2] V. Chaudhuri et al, (2008). Functions of Varicella-zoster virus ORF23 capsid protein in viral replication and the pathogenesis of skin infection. J Virol 82(20), 10231-46.

[3] P. J. Skinner et al, (1997). Ataxin-1 with an expanded glutamine tract alters nuclear matrixassociated structures. Nature 389(6654), 971-4.

[4] R. D. Everett and M. K. Chelbi-Alix, (2007). PML and PML nuclear bodies: implications in antiviral defence. Biochimie 89(6-7), 819-30.

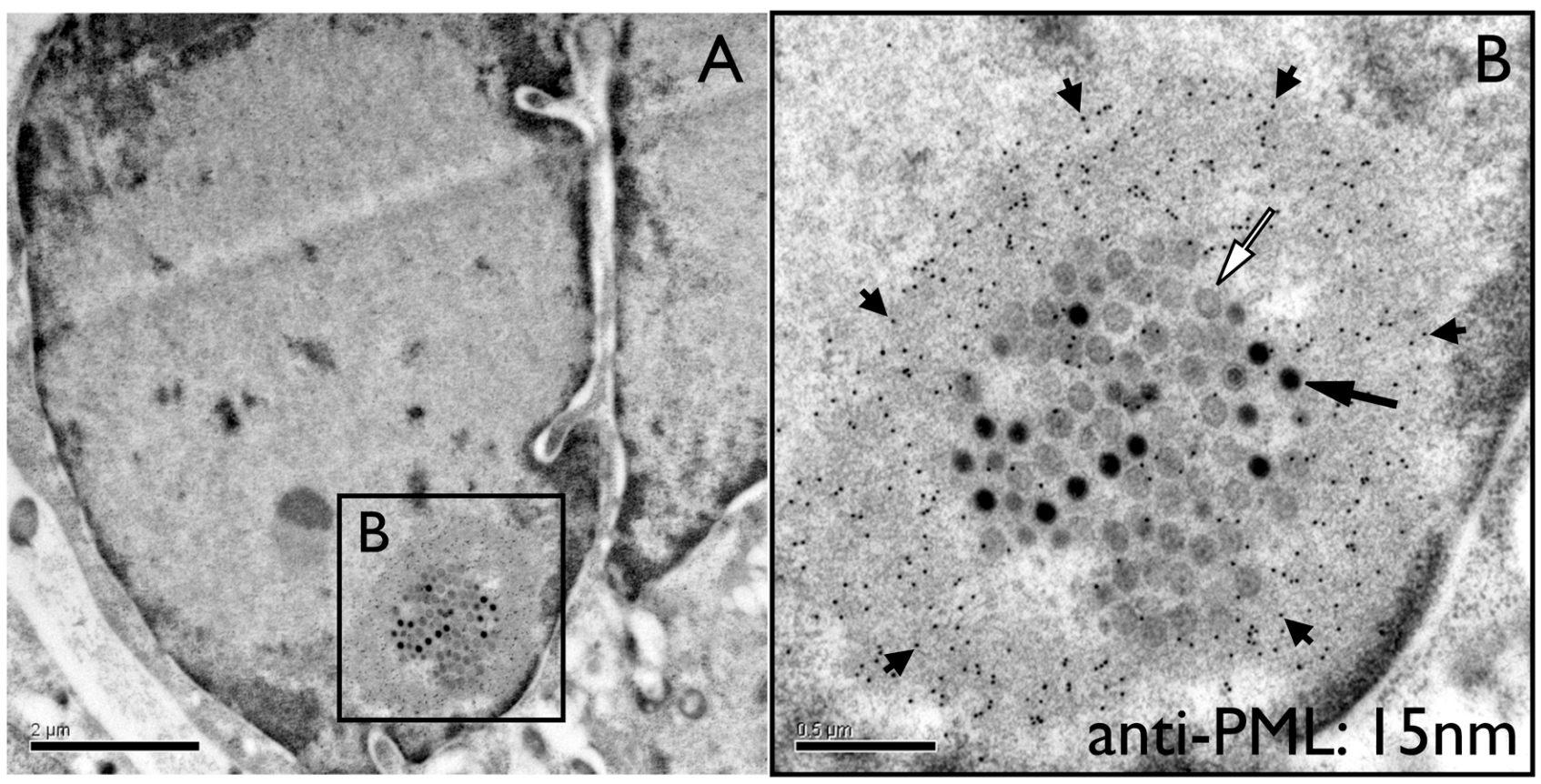

FIG.1. Spherical PML-IV cages sequester Varicella-zoster virus capsids within the cell nucleus. VZV-infected cells were high-pressure frozen, freeze-substituted and embedded in LR-White resin. Ultrathin sections were immuno-gold labeled with a polyclonal (rabbit) anti-PML antibody and $15 \mathrm{~nm}$ protein A-gold particles. (A) Overview of an VZV-infected cell nucleus. Area B is shown at higher magnification on the right. (B) A spherical PML-IV cage is densley labeled for PML (small arrowheads depict PML-specific gold particels). Mature viral capsids (dark arrow) and intermediate capsids (white arrow) are indicated. 